\title{
AN ANALYSIS OF TAGUCHI'S ON-LINE QUALITY MONITORING PROCEDURE FOR VARIABLES BASED ON THE RESULTS OF A SEQUENCE OF INSPECTIONS
}

\author{
Linda Lee $\mathrm{Ho}^{1 *}$ and Roberto Quinino ${ }^{2}$
}

Received March 11, 2010 / Accepted November 12, 2011

\begin{abstract}
In this paper, a procedure for the on-line process control of variables is proposed. This procedure consists of inspecting the $m$-th item from every $m$ produced items and deciding, at each inspection, whether the process is out-of-control. Two sets of limits, warning $\left(\mu_{0} \pm W\right)$ and control $\left(\mu_{0} \pm C\right)$, are used. If the value of the monitored statistic falls beyond the control limits or if a sequence of $h$ observations falls between the warning limits and the control limits, the production is stopped for adjustment; otherwise, production goes on. The properties of an ergodic Markov chain are used to obtain an expression for the average cost per item. The parameters (the sampling interval $m$, the widths of the warning, the control limits $W$ and $C(W \leq C)$, and the sequence length $(h)$ are optimized by minimizing the cost function. A numerical example illustrates the proposed procedure.
\end{abstract}

Keywords: on-line process control, quality control by variables, Markov chain, warning limit, sequence of inspections.

\section{INTRODUCTION}

Taguchi et al. $(1985,1989)$ proposed an economical procedure to monitor on-line process control with attributes as well as variables. This inspection system is automatic and allows for the sampling of only a single item at a time. In general, the proposed system can be implemented in high-speed electronics manufacturing facilities, where the testing equipment is connected to a central computer where data are taken automatically as the items that are produced are tested.

The procedure of on-line control for use in monitoring processes has been studied by many authors, such as Adams \& Woodall (1989), Nayebpour \& Woodall (1993), Srivastava \& Wu (1991, 1995), Gong \& Tang (1997), Borges et al. (2001), Wang \& Yue (2001), Dasgupta (2003), Trindade et al. (2007), Dasgupta \& Mandal (2008), Ho et al. (2007), Trindade et al. (2007) and Quinino et al. (2010). Ho \& Trindade (2009) presented a revision related to this topic and proposed an economical X control chart model for short-run production.

\footnotetext{
*Corresponding author

${ }^{1}$ Universidade de São Paulo, Departamento de Engenharia de Produção - Escola Politécnica. E-mail: lindalee@usp.br

${ }^{2}$ Universidade Federal de Minas Gerais, Departamento de Estatística - ICEX. E-mail: roberto@est.ufmg.br
} 
In Taguchi's approach [Taguchi et al. $(1989,2004)]$, initially the process mean is $\mu=\mu_{0}$ (state I). At a random time, the process mean may shift from $\mu=\mu_{0}$ to $\mu=\mu_{1}>\mu_{0}$ (state II). To control the quality of the process, after every $m$ items have been produced, the $m$-th item is examined. If the value of the quality characteristic does not satisfy the control limit, then production is stopped for adjustment. However, Taguchi et al. (1989; 2004) did not assume explicitly a probability function for the shift of the process mean from $\mu=\mu_{0}$ to $\mu=\mu_{1}>$ $\mu_{0}$, and many simplifications and approximations were used to calculate the average cost per produced item and the optimum values of the sampling interval $m$ (hereafter denoted as $m^{\circ}$ ) and the width of the control limit $C$.

Adams \& Woodall (1989) argued the inadequacy of Taguchi's model and presented alternative procedures for random walk models. The optimum sampling interval and control limits obtained by direct search are presented in tables. Srivastava \& Wu $(1991,1995)$ presented a second order approximation for Taguchi's loss function and therefore presented closed expressions for the optimum sampling interval and control limits were also yielded.

Traditionally, three horizontal lines are used to draw the standard (Shewhart) control charts: a central line (representing the average value of the quality characteristic at an in-control state) and two other horizontal lines, called the control limits (upper and lower). However, some analysts may suggest two sets of limits. The outer limits are the usual action limits and are called control limits (CL) (points that plot outside of this limit lead to a stoppage for an assignable cause) and the inner limits are called warning limits (WL). Points that fall between the warning limits and the control limits may cause suspicion that the process may not be operating properly. One may also suggest for an additional rule, similar to the additional rules described in the Western Electric Handbook (1956) to decide if the production process is in-control or not. Such additional rules are usually based on the sequence of the results of last inspections.

The purpose of this paper is to present a procedure for on-line process control by adding alternative criteria that lead to an interruption of the production process not included in the previous listed papers. As in Taguchi's approach, the $m$-th item is inspected at every $m$ produced items. If the value of the monitored statistic falls beyond the control limits (red zone - RZ) or a sequence of $h$ observations falls between the warning limits and the control limits (yellow zone - YZ), then the production is stopped for adjustment; otherwise, (green zone-GZ) production goes on. The parameters $h, C, W$ and $\mathrm{m}$ are determined such that they minimize the average cost per item of the controlled system. Figure 1 represents schematically the new proposal.

This paper expands the procedure proposed by Ho et al. (2007). Their contribution can be viewed as a specific case of the new proposal, when $h=1$ and $W=C$. The generalization proposed in the current paper, $h \geq 1$ and $W \leq C$, does not constitute a simple methodological expansion of Ho et al.'s proposal. A new development, based on the Markov chain, is required to facilitate the advancements and to provide better understanding for the reader. Additionally, restrictions related to the minimum value of the in-control average run length $\left(A R L_{0}\right)$ and a maximum value of the average run length during an out-of-control condition $\left(A R L_{\varepsilon}\right)$ are included in the optimization process. A comparison of the results from the current proposal with the results 


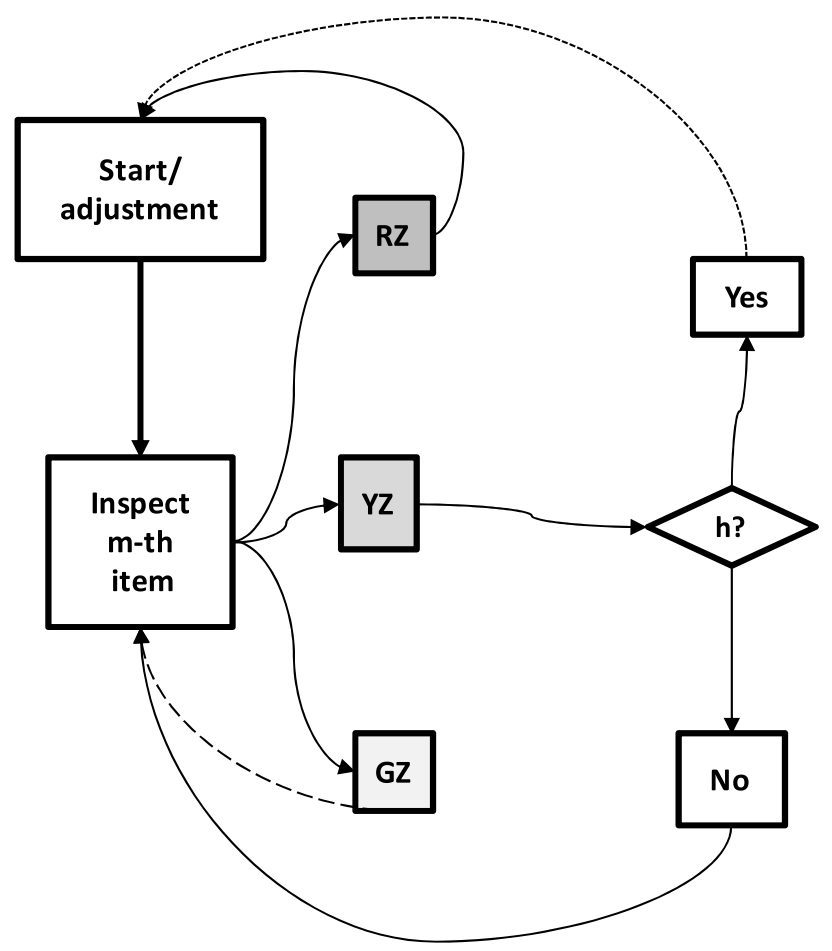

Figure 1 - Schematic representation of the inspection system.

obtained by Ho et al. (2007) is successful. This paper is organized as follows: Section 2 describes the probabilistic inspection model. In Section 3, an economic model is developed to determine the optimum designs. In Section 4, a numerical example illustrates the proposed model. The conclusions are presented in Section 5.

\section{PROBABILISTIC MODEL}

Consider a process that produces items and a quality characteristic of interest $(X)$ that can be described by a Normal distribution with mean $\mu$ and standard deviation $\sigma$. The process starts in-control, in other words, its process mean is $\mu=\mu_{0}$ (State I), and the process may shift to $\mu=\mu_{1}, \mu_{1} \neq \mu_{0}$. The duration of the process in-control condition is usually modeled by an exponential distribution for a continuous case. The geometric distribution behaves similarly to the exponential distribution, but it is typically used for the discrete case in which the duration is measured by the number of units that are produced before the shift. Following previous papers [Nayebpour \& Woodall (1993), Nandi \& Sreehari (1999), Jiang \& Tsui (2000), Borges et al. (2001), Ho et al. (2007), Trindade et al. (2007), Dasgupta (2008) and Ding \& Gong (2008)], this study relies on a geometric distribution with parameter $\pi, 0<\pi<1$, to describe the random shift from State I to State II. One assumes that the probability of shifts from State II to State I is zero without any intervention in the process. 
Both the geometric and exponential distributions are memoryless, which facilitates mathematical analysis. However, these distributions are useful for other reasons than their mathematical facility. Many researchers have recently used exponential or geometric distributions to describe shifts from in-control to out-of-control states. For example, Wang \& Sheu (2003), Zhang et al. (2008), Serel \& Moskowistz (2008), and Lim \& Cho (2009) employed exponential distributions, but Ho et al. (2007), Trindade et al. (2007), Dasgupta (2008) and Ding \& Gong (2008) used geometric distributions. Such distributions not only facilitate the development of mathematical models but also allow application to real problems, as mentioned in the cited papers.

Three horizontal lines are used to build standard (Shewhart) control charts, one drawn at $\mu=\mu_{0}$ and two others at $\mu_{0} \pm C$ (upper and lower control limits (CL)). However, one may suggest two sets of limits; the outer limits are the usual action limits (CL), and the inner limits are drawn at $\mu_{0} \pm W, W \leq C$. To illustrate these limits (see Fig. 2), one may use different colors to identify the different zones. Points that plot outside of the control limits lead to a stoppage for an assignable cause (red zone); points that fall between the warning limits and the control limits may reveal that the process may not be operating adequately (yellow zone); otherwise, the process must operate adequately if the points fall between the warning limits (green zone).

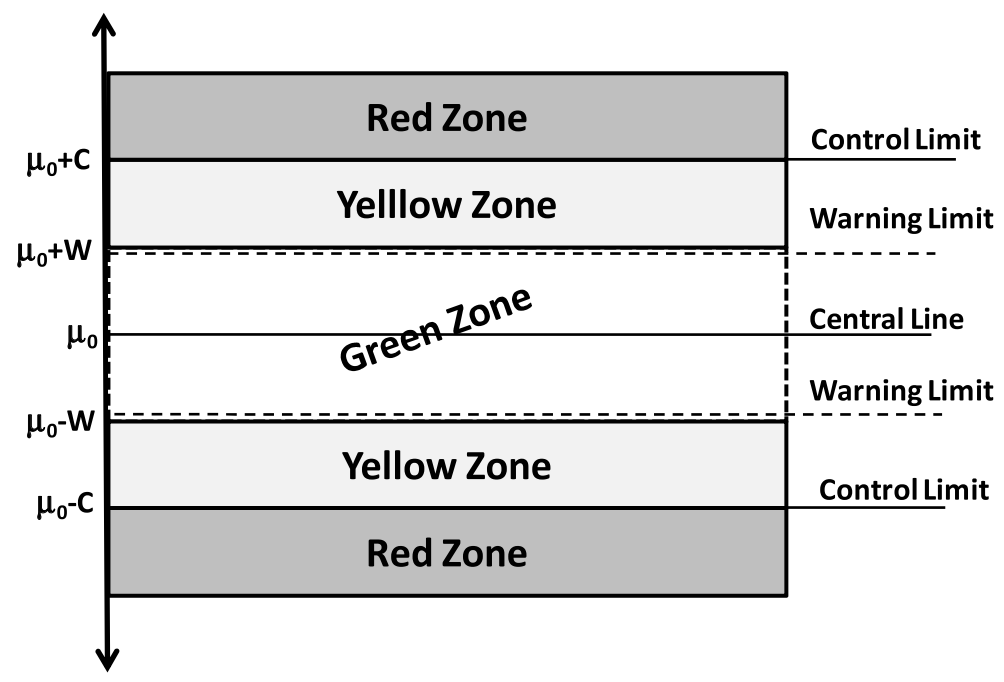

Figure 2 - Warning and control limits.

Let $X_{i}, i=1,2,3, \ldots, \infty$, denote the observed values of some characteristic of interest at the $i$-th inspection. The system of inspection may be described by a Markov chain with a set of states $(s, k)$. The first index indicates in which state [State I: in-control $(s=0)$ or State II: outof-control $(s>0)]$ the process is at the moment of inspection and assumes three values: $s=0$, the inspected item is produced at $\mu=\mu_{0} ; s=1$, the inspected item is produced at $\mu=\mu_{1}$, but the process mean shifts from $\mu=\mu_{0}$ to $\mu=\mu_{1}$ in the current inspection (thus, a portion of the items is produced at $\mu=\mu_{0}$, and at least the inspected item is produced at $\left.\mu=\mu_{1}\right) ; s=2$, the 
process mean has shifted to $\mu=\mu_{1}$, and all of the items in the current inspection are produced at $\mu=\mu_{1}$. The second index $k$ is related to the result of the inspection:

- $k=-1$, the observed value of the current inspection is in the red zone $\left(x_{i}>\mu_{0}+C\right.$ or $x_{i}<\mu_{0}-C$ ); the next inspection is only after a production of $m$ items, and the process is stopped for adjustment;

- $k=0$, the observed value is in the green zone, $\left(\mu_{0}-W \leq X_{i} \leq \mu_{0}+W\right)$ and the process is not adjusted;

- $k=1$, the observed value is in the yellow zone $\left(\mu_{0}+W \leq X_{i}<\mu_{0}+C\right.$ or $\mu_{0}-C<$ $\left.X_{i} \leq \mu_{0}-W\right)$, but the value of the previous inspection was not in the yellow zone and the process is not adjusted;

- $k=2$, the observed value is in the yellow zone $\left(\mu_{0}+W \leq X_{i}<\mu_{0}+C\right.$ or $\mu_{0}-C<$ $\left.X_{i} \leq \mu_{0}-W\right)$, like the previous inspection and the process is not adjusted;

- $k=3$, the observed value is in the yellow zone $\left(\mu_{0}+W \leq X_{i}<\mu_{0}+C\right.$ or $\mu_{0}-C<$ $\left.X_{i} \leq \mu_{0}-W\right)$, like the two previous inspections and the process is not adjusted;

- $k=h$, the observed value is in the yellow zone $\left(\mu_{0}+W \leq X_{i}<\mu_{0}+C\right.$ or $\mu_{0}-C<$ $\left.X_{i} \leq \mu_{0}-W\right)$, like the $(h-1)$ previous inspections; the process is adjusted.

Note that there is an adjustment only if $k=-1$ or $k=h$. A total of $3(h+2)$ states of chain $(s, k)$ is used to describe the inspection process. The second index assumes integer values in the interval $-1 \leq k \leq h$ and $k=t \geq 0$, which indicates a sequence of $t$ inspected items for which the observed values are in the yellow zone. Before the probabilistic model, let us introduce the following notations:

$$
\begin{aligned}
G_{0} & =P\left(\mu_{0}-W \leq X_{i} \leq \mu_{0}+W \mid \mu=\mu_{0}\right) \\
G_{1} & =P\left(\mu_{0}-W \leq X_{i} \leq \mu_{0}+W \mid \mu=\mu_{1}\right) \\
Y_{0} & =P\left(\mu_{0}+W \leq X_{i}<\mu_{0}+C \mid \mu=\mu_{0}\right)+P\left(\mu_{0}-C<X_{i} \leq \mu_{0}-W \mid \mu=\mu_{0}\right) \\
Y_{1} & =P\left(\mu_{0}+W \leq X_{i}<\mu_{0}+C \mid \mu=\mu_{1}\right)+P\left(\mu_{0}-C<X_{i} \leq \mu_{0}-W \mid \mu=\mu_{1}\right) \\
R_{0} & =P\left(X_{i}>\mu_{0}+C \mid \mu=\mu_{0}\right)+P\left(X_{i}<\mu_{0}-C \mid \mu=\mu_{0}\right) \\
R_{1} & =P\left(X_{i}>\mu_{0}+C \mid \mu=\mu_{1}\right)+P\left(X_{i}<\mu_{0}-C \mid \mu=\mu_{1}\right)
\end{aligned}
$$

The transition probabilities from state $(s, k)$ at the moment of inspection $i$ to state $\left(s^{*}, k^{*}\right)$ at the moment of inspection $(i+1)$ are elements of the matrix $\mathbf{P}$

$$
\mathbf{P}=\left(\begin{array}{lll}
\mathbf{A}_{00} & \mathbf{A}_{01} & \mathbf{A}_{02} \\
\mathbf{A}_{10} & \mathbf{A}_{11} & \mathbf{A}_{12} \\
\mathbf{A}_{20} & \mathbf{A}_{21} & \mathbf{A}_{22}
\end{array}\right)
$$


where $\mathbf{A}_{\mathbf{s s}}$ denotes the matrix of transitory probabilities $p_{(s, k)(s *, k *)} ; s, s^{*}=0,1,2 ; k$, $k^{*}=-1,0, \ldots, h$. The elements of $\mathbf{A}_{\mathbf{0 0}}$ are the transitory probabilities from states $(0, k)$ to states $\left(0, k^{*}\right)$. That is, the process is in-control at the $i$-th inspection, and it also stays in-control at the $(i+1)$-th inspection. The non-null elements of the matrix $\mathbf{A}_{\mathbf{0 0}}$ are expressed in (2).

$$
\left\{\begin{array}{l}
p_{(0,0)(0,-1)}=p_{(0,-1)(0,-1)}=p_{(0, h)(0,-1)}=p_{(0, k)(0,-1)=q_{m} R_{0}} \\
p_{(0,0)(0,0)}=p_{(0,-1)(0,0)}=p_{(0, h)(0,0)}=p_{(0, k)(0,0)=q_{m} G_{0}} \\
p_{(0,0)(0,1)}=p_{(0,-1)(0,1)}=p_{(0, h)(0,1)}=p_{(0, k)(0, k+1)=q_{m} Y_{0}}
\end{array}\right.
$$

for $0<k<h$, with $q_{m}=(1-\pi)^{m}$.

The elements of $\mathbf{A}_{01}$ are the transition probabilities from states $(0, k)$ to states $\left(1, k^{*}\right)$. That is, the process is in control at the $i$-th inspection, but the parameter shifts from $\mu=\mu_{0}$ to $\mu=\mu_{1}$. Thus, some items are produced at $\mu=\mu_{0}$, and at least the inspected item is produced at $\mu=\mu_{1}$. The non-null elements of the matrix $\mathbf{A}_{\mathbf{0 1}}$ are expressed in (3), as follows:

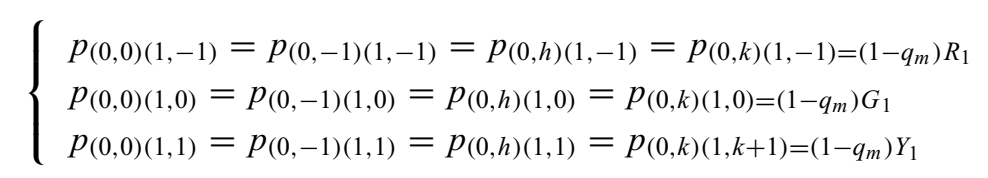

for $0<k<h$, with $q_{m}=(1-\pi)^{m}$.

The matrix $\mathbf{A}_{\mathbf{0 2}}$ is a null matrix; $\mathbf{A}_{\mathbf{1 0}}=\mathbf{A}_{\mathbf{2 0}}$ and their non-null elements are the transitory probabilities with $k=-1$ or $h$. That is, the process is adjusted in the previous inspection, and it restarts in-control in the current inspection employing the sampling interval $m$ :

$$
\left\{\begin{array}{l}
p_{(1,-1)(0,-1)}=p_{(2,-1)(0,-1)}=p_{(1, h)(0,-1)}=p_{(2, k)(0,-1)=q_{m} R_{0}} \\
p_{(1,-1)(0,0)}=p_{(2,-1)(0,0)}=p_{(1, h)(0,0)}=p_{(2, h)(0,0)=q_{m} G_{0}} \\
p_{(1,-1)(0,1)}=p_{(2,-1)(0,1)}=p_{(1, h)(0,1)}=p_{(2, h)(0,1)=q_{m} Y_{0}} .
\end{array}\right.
$$

Similarly, the non-null elements of $\mathbf{A}_{\mathbf{1 1}}=\mathbf{A}_{\mathbf{2 1}}$ are also transition probabilities with $k=-1$ or $h$. However, after the adjustment, the process restarts in-control, but the parameter $\mu$ shifts in the current inspection. Thus, the non-null elements of these matrices are

$$
\left\{\begin{array}{l}
p_{(1,-1)(1,-1)}=p_{(2,-1)(1,-1)}=p_{(1, h)(1,-1)}=p_{(2, h)(1,-1)=\left(1-q_{m}\right) R_{1}} \\
p_{(1,-1)(1,0)}=p_{(2,-1)(1,0)}=p_{(1, h)(1,0)}=p_{(2, h)(1,0)=\left(1-q_{m}\right) G_{1}} \\
p_{(1,-1)(1,1)}=p_{(2,-1)(1,1)}=p_{(1, h)(1,1)}=p_{(2, h)(1,1)=\left(1-q_{m}\right) Y_{1}}
\end{array}\right.
$$

Next, we need the matrices $\mathbf{A}_{12}$ and $\mathbf{A}_{\mathbf{2 2}}$, which are the transition probabilities from states $(1, k)$ to states $\left(2, k^{*}\right)$ and from states $(2, k)$ to states $\left(2, k^{*}\right)$, respectively. In these cases, the parameter shifted in the previous inspections, and the process has not been adjusted. $\mathbf{A}_{\mathbf{1 2}}=\mathbf{\mathbf { A } _ { 2 2 }}$ and the non-null probabilities of these matrices are

$$
\left\{\begin{array}{l}
p_{(1, k)(2,-1)}=p_{(2, k)(2,-1)}=R_{1} \\
p_{(1, k)(2,0)}=p_{(2, k)(2,0)}=G_{1} \\
p_{(1, k)(2, k+1)}=p_{(2, k)(2, k+1)}=Y_{1}
\end{array}\right.
$$


for $0 \leq k<h$. Note that the Markov chain built in this way incorporates information about the process (whether it is in-control or out-of-control), the length of the sequence of observations in the yellow zone and also the length of sampling intervals $m$ in the matrix $\mathbf{P}$. Matrix $\mathbf{P}$ is ergodic recurrent and consequently $\boldsymbol{\Delta}=\lim _{u \rightarrow \infty} \mathbf{P}^{u}$ exists and does not depend on the probability of the initial states of the process. Thus, all rows of $\boldsymbol{\Delta}$ are equal. By denoting the first row of the matrix $\boldsymbol{\Delta}$ by $\delta=\left(\delta_{(0,-1)}, \ldots, \delta_{(0, h)}, \delta_{(1,-1)}, \ldots, \delta_{(1, h)}, \delta_{(2,-1)}, \ldots, \delta_{(2, h)}\right)$ and solving the system of linear equations $\boldsymbol{\delta}=\boldsymbol{\delta} \mathbf{P}$ subject to the restriction $\sum_{s=0}^{2} \quad \sum_{k=-1}^{h} \delta_{(s, k)}=1, \delta_{(s, k)}$ can be determined. This solution can be interpreted as the proportion of inspections in state $(s, k)$, $s=0,1,2 ; k=-1,0, \ldots, h$ over a large number of inspections.

\section{COST FUNCTION}

To obtain the cost function, more assumptions are needed. Once one decides to make an adjustment, the stoppage of the process is instantaneous. After the adjustment, the process restarts at State I $\left(\mu=\mu_{0}\right)$. In this paper, the costs follow a structure similar to the usual economic designs, where $c_{i}$ is the cost for a single inspection and $c_{a}$ is the cost for adjustment. In this paper, one item is stated as non-conforming if the observed value of the quality characteristic is beyond the specification limits $\left(\mu_{0} \pm L E\right)$. Thus, the next costs related to non-conforming and discarded items are also included in the model: $c_{n}$ is the cost for delivering a non-conforming item (this item is sent to the customer or to the following production stage), and $c_{D}$ is the cost to discard the examined item. For each cycle of inspection, the $(m-1)$ items are sent to the customer or to the following stages of production. It is assumed that all inspected items are discarded or rectified in the posterior stages once the inspection is partially destructive.

For a sufficiently large number of inspections,

$$
\boldsymbol{\delta}=\left(\delta_{(0,-1)}, \ldots, \delta_{(0, h)}, \delta_{(1,-1)}, \ldots, \delta_{(1, h)}, \delta_{(2,-1)}, \ldots, \delta_{(2, h)}\right)
$$

is the vector of the probability of the states of the Markov chain. Let $C$ be the random variables related to the cost at each cycle of inspection. We assume discrete values related to the states of the Markov chain. The cost of the state $(s, k), s=0,1,2 ; k=-1,0, \ldots, h$ may be written as

$$
C(s, k)=c_{i}+a(s, k)+n(s, k)+c_{D} ; s=0,1,2 ; k=-1, \ldots, h
$$

- $c_{i}, c_{D}$, respectively, are the costs to inspect and discard a single item, constants for all states $(s, k), s=0,1,2 ; k=-1,0, \ldots, h$;

- $a(s, k)$ is the cost to adjust the process;

- $n(s, k)$ is the cost to send non-conforming items to the customer or to later stages of the process.

In the next paragraphs, the different costs are detailed.

- Cost to adjust the process: 
The cost for adjustment is included for states $(s, k), s=0,1,2 ; k=-1, h$. Thus,

$$
a(s, k)=c_{a} ; s=0,1,2 ; k=-1, h .
$$

- The cost to send non-conforming items to the customer or to later stages of the process:

For the states $(0, k), k=-1,0, \ldots, h$, all items are produced at $\mu=\mu_{0}$; thus,

$$
n(0, k)=c_{n} p_{1}(m-1) ; k=-1,0, \ldots, h,
$$

with $p_{1}=1-P\left(\mu_{0}-L E \leq X_{i} \leq \mu_{0}+L E \mid \mu=\mu_{0}\right)$. Similarly for the states $(2, k)$, when all items are produced at $\mu=\mu_{1}$, we have

$$
n(2, k)=c_{n} p_{2}(m-1) ;
$$

for $k=-1,0, \ldots, h$, with $p_{2}=1-P\left(\mu_{0}-L E \leq X_{i} \leq \mu_{0}+L E \mid \mu=\mu_{1}\right)$. For the states $(1, k), k=-1,0, \ldots, h, v<m$ items are produced at $\mu=\mu_{0}$, and then, $m-v$ are produced at $\mu=\mu_{1}$. Taking into account all possibilities

$$
n(1, k)=c_{n} \frac{\sum_{v=1}^{n} \pi(1-\pi)^{v-1}\left[(v-1) p_{1}+(m-v) p_{2}\right]}{1-(1-\pi)^{m}}
$$

for $k=-1,0, \ldots, h$. For a large number of inspections and considering our setting as a renewal-reward process, the average cost per item $C E(m)$ is the ratio of the expected cost per inspection cycle $E(V)$ by the quantity of items sent to the customer or to the following stages of production, expressed as

$$
C E(m)=\frac{E(V)}{m-1}=\frac{\sum_{s=0}^{2} \sum_{k=-1}^{h} C(s, k) \delta_{(s, k)}}{m-1} .
$$

Montgomery (2001) claimed that the economical design of the control chart must be evaluated jointly with some statistical criteria. He recommended strongly that the optimization of the cost function should be subject to an adequate statistical restriction. In this study, a set of additional restrictions are considered: a minimum value for the in-control average run length $\left(A R L_{0}\right)$ is denoted by $\phi_{0}$, and a maximum value for the average run length during an out-of-control condition $\left(A R L_{\varepsilon}\right)$ is denoted by $\phi_{1}$. These values $\left(\phi_{0}\right.$ and $\left.\phi_{1}\right)$ are fixed as a part of the responsibility for the control system of the productive process.

The problem consists of determining the values of

$$
\begin{aligned}
\left(m^{\circ}, W^{\circ}, C^{\circ}, h^{\circ}\right)= & \underset{(m, W, C, h)}{\arg \min }[C E(m)] \\
& \text { subject to }\left\{\begin{array}{l}
A R L_{0} \geq \phi_{0}, \\
A R L_{\varepsilon} \leq \phi_{1}
\end{array}\right.
\end{aligned}
$$

with $m$ the sampling interval; the constants $W$ and $C$ are used to draw, respectively, the warning limit and the control limit, and $h$ is the length of the sequence of values that are in the yellow zone. 
The values of $A R L_{0}, A R L_{\varepsilon}$ can be calculated by employing the stationary vector

$$
\begin{aligned}
\delta= & \left(\delta_{(0,-1)}, \delta_{(0,0)}, \ldots, \delta_{(0, h-1)}, \delta_{(0, h)}, \delta_{(1,-1)}, \delta_{(1,0)}, \ldots\right. \\
& \left.\delta_{(1, h-1)}, \delta_{(1, h)}, \delta_{(2,-1)}, \delta_{(2,0)}, \ldots, \delta_{(2, h-1)}, \delta_{(2, h)}\right)
\end{aligned}
$$

and can be expressed as:

$$
\begin{aligned}
& A R L_{0}=\left(\frac{\delta_{(0,-1)}+\delta_{(0, h)}}{\delta_{(0,-1)}+\cdots+\delta_{(0, h)}}\right)^{-1} \\
& A R L_{\varepsilon}=\left(\frac{\delta_{(1,-1)}+\delta_{(1, h)}+\delta_{(2,-1)}+\delta_{(2, h)}}{\delta_{(1,-1)}+\cdots+\delta_{(1, h)}+\delta_{(2,-1)}+\cdots+\delta_{(2, h)}}\right)^{-1}
\end{aligned}
$$

\section{NUMERICAL EXAMPLES AND DISCUSSIONS}

To illustrate the proposed procedure, consider the example adapted from Taguchi et al. (1989), Taguchi et al. (2004) and Trindade et al. (2007).

A manufacturer of high-volume-integrated circuits wants to install a system to control the measurement of some dimension of interest. Historical data allows an estimation of the cost components as $c_{i}=\$ 0.25, c_{n}=\$ 20, c_{D}=\$ 2.0$, and $c_{a}=\$ 900$. The specification limits (LE) were fixed as \pm 1.5 , and the shifts from process in-control [State I $\left(\mu_{0}=0\right)$ ] to out-of-control [State II $\left(\mu_{1}=1\right)$ ] can be described by a geometric distribution with the parameter $\pi=0.001$. The standard deviation of the process is known and is equal to 0.5 . Managers responsible for the production system adopt $\phi_{0}=370$ and $\phi_{1}=5$ (minimum and maximum values for $A R L_{0}$ and $A R L_{\varepsilon}$, respectively). To calculate the optimum values, a program in MatLab was developed for this task. (Interested readers can request a copy of the program directly from the authors.)

Figure 3 shows the plots of the expected cost versus the sampling interval $m$ for some cases chosen to illustrate the behavior of the optimum set. The optimal design is as follows: The sampling interval is $m^{0}=27$. The length of the sequence of observations in the yellow zone is $h^{0}=3$. The width of the warning limit is $W^{0}=0.8$, and the width of the control limit is $C^{0}=1.6$; the results yield an average cost of $\$ 1.381$. The current proposal is $4.4 \%$ less expensive when compared with the approach presented in Ho, Medeiros \& Borges (2007). In that case, the sampling interval increases to $m^{0}=32$ as the average cost also increases to $\$ 1.445$ (with $h=1$ and $W=C=1.4$ ).

\subsection{Sensitivity analysis}

For the sensitivity analysis, each parameter is analyzed in a $\pm 15 \%$ range of values, and other parameters are kept equal to the numerical values presented at the beginning of this section.

Table 1 summarizes the results. As expected, increases in the cost $c_{n}$ and the parameters $\mu_{1}$ and $\pi$ yield a decrease in the values of the sampling interval $m$ (and vice versa). The values of $h, W$ and $C$ are not sensible in the range of variation up to $15 \%$ in the parameters of the process and 


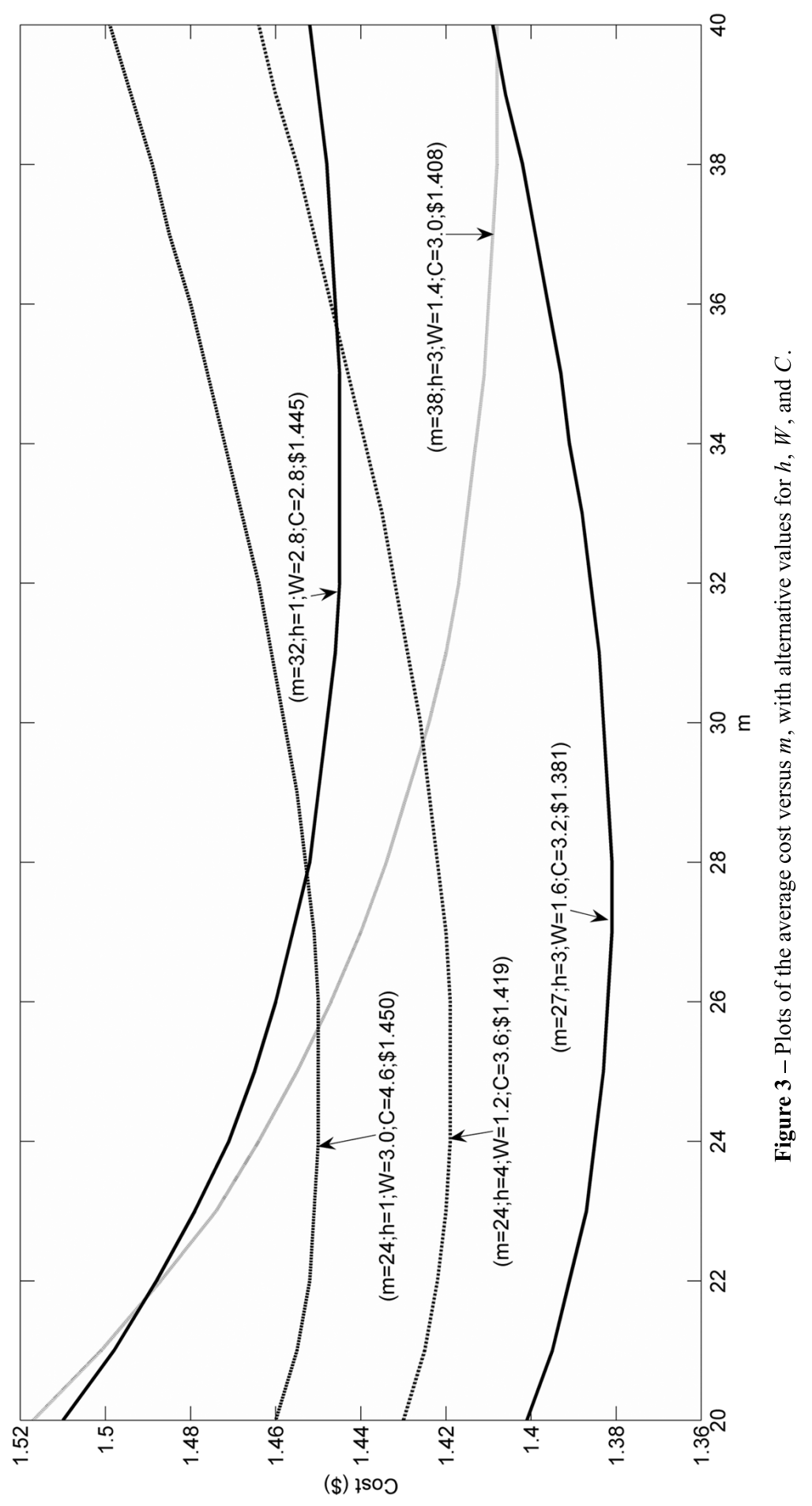

Pesquisa Operacional, Vol. 32(1), 2012 
Table 1 - Sensitivity analysis of the cost components and parameters.

\begin{tabular}{|c|c|c|c|c|c|c|c|c|c|c|c|c|c|}
\hline & $c_{n}$ & $\mathrm{~m}$ & Cost & w & C & h & & $\mathrm{c}_{\mathrm{a}}$ & $\mathrm{m}$ & Cost & W & C & $\mathrm{h}$ \\
\hline \multirow{12}{*}{$c_{n}$} & 17.00 & 31 & 1.32 & 0.80 & 1.60 & 3 & \multirow{11}{*}{$\mathrm{c}_{\mathrm{a}}$} & 765 & 28 & 1.24 & 1.00 & 1.60 & 2 \\
\hline & 17.60 & 30 & 1.33 & 0.80 & 1.60 & 3 & & 792 & 28 & 1.27 & 1.00 & 1.60 & 2 \\
\hline & 18.20 & 29 & 1.35 & 0.80 & 1.60 & 3 & & 819 & 26 & 1.30 & 0.80 & 1.60 & 3 \\
\hline & 18.80 & 29 & 1.36 & 0.80 & 1.60 & 3 & & 846 & 26 & 1.33 & 0.80 & 1.60 & 3 \\
\hline & 19.40 & 28 & 1.37 & 0.80 & 1.60 & 3 & & 873 & 27 & 1.35 & 0.80 & 1.60 & 3 \\
\hline & 20.00 & 27 & 1.38 & 0.80 & 1.60 & 3 & & 900 & 27 & 1.38 & 0.80 & 1.60 & 3 \\
\hline & 20.60 & 27 & 1.39 & 0.80 & 1.60 & 3 & & 927 & 28 & 1.41 & 0.80 & 1.60 & 3 \\
\hline & 21.20 & 26 & 1.40 & 0.80 & 1.60 & 3 & & 954 & 28 & 1.44 & 0.80 & 1.60 & 3 \\
\hline & 21.80 & 26 & 1.42 & 0.80 & 1.60 & 3 & & 981 & 29 & 1.46 & 0.80 & 1.60 & 3 \\
\hline & 22.40 & 25 & 1.43 & 0.80 & 1.60 & 3 & & 1008 & 29 & 1.49 & 0.80 & 1.60 & 3 \\
\hline & 23.00 & 25 & 1.44 & 0.80 & 1.60 & 3 & & 1035 & 29 & 1.52 & 0.80 & 1.60 & 3 \\
\hline & $C_{D}$ & $\mathrm{~m}$ & Cost & W & $C$ & $\mathrm{~h}$ & & $c_{i}$ & $\mathrm{~m}$ & Cost & W & $C$ & $\mathrm{~h}$ \\
\hline \multirow{12}{*}{$C_{D}$} & 1.70 & 26 & 1.37 & 0.80 & 1.60 & 3 & \multirow{12}{*}{$c_{i}$} & 0.21 & 27 & 1.38 & 0.80 & 1.60 & 3 \\
\hline & 1.76 & 27 & 1.37 & 0.80 & 1.60 & 3 & & 0.22 & 27 & 1.38 & 0.80 & 1.60 & 3 \\
\hline & 1.82 & 27 & 1.37 & 0.80 & 1.60 & 3 & & 0.23 & 27 & 1.38 & 0.80 & 1.60 & 3 \\
\hline & 1.88 & 27 & 1.38 & 0.80 & 1.60 & 3 & & 0.24 & 27 & 1.38 & 0.80 & 1.60 & 3 \\
\hline & 1.94 & 27 & 1.38 & 0.80 & 1.60 & 3 & & 0.24 & 27 & 1.38 & 0.80 & 1.60 & 3 \\
\hline & 2.00 & 27 & 1.38 & 0.80 & 1.60 & 3 & & 0.25 & 27 & 1.38 & 0.80 & 1.60 & 3 \\
\hline & 2.06 & 27 & 1.38 & 0.80 & 1.60 & 3 & & 0.26 & 27 & 1.38 & 0.80 & 1.60 & 3 \\
\hline & 2.12 & 28 & 1.39 & 0.80 & 1.60 & 3 & & 0.27 & 27 & 1.38 & 0.80 & 1.60 & 3 \\
\hline & 2.18 & 28 & 1.39 & 0.80 & 1.60 & 3 & & 0.27 & 27 & 1.38 & 0.80 & 1.60 & 3 \\
\hline & 2.24 & 28 & 1.39 & 0.80 & 1.60 & 3 & & 0.28 & 27 & 1.38 & 0.80 & 1.60 & 3 \\
\hline & 2.30 & 31 & 1.39 & 1.00 & 1.60 & 3 & & 0.29 & 27 & 1.38 & 0.80 & 1.60 & 3 \\
\hline & $\mu_{1}$ & $\mathrm{~m}$ & Cost & $w$ & $C$ & $h$ & & $\pi\left(\times 10^{-3}\right)$ & $\mathrm{m}$ & Cost & w & $C$ & $\mathrm{~h}$ \\
\hline \multirow{11}{*}{$\mu_{1}$} & 0.85 & 35 & 1.29 & 0.80 & 1.60 & 3 & \multirow{11}{*}{$\pi\left(\times 10^{-3}\right)$} & 85 & 28 & 1.22 & 0.80 & 1.60 & 3 \\
\hline & 0.88 & 33 & 1.31 & 0.80 & 1.60 & 3 & & 88 & 28 & 1.26 & 0.80 & 1.60 & 3 \\
\hline & 0.91 & 31 & 1.33 & 0.80 & 1.60 & 3 & & 91 & 28 & 1.29 & 0.80 & 1.60 & 3 \\
\hline & 0.94 & 29 & 1.35 & 0.80 & 1.60 & 3 & & 94 & 27 & 1.32 & 0.80 & 1.60 & 3 \\
\hline & 0.97 & 28 & 1.37 & 0.80 & 1.60 & 3 & & 97 & 27 & 1.35 & 0.80 & 1.60 & 3 \\
\hline & 1.00 & 27 & 1.38 & 0.80 & 1.60 & 3 & & 100 & 27 & 1.38 & 0.80 & 1.60 & 3 \\
\hline & 1.03 & 26 & 1.39 & 0.80 & 1.60 & 2 & & 103 & 27 & 1.41 & 0.80 & 1.60 & 3 \\
\hline & 1.06 & 26 & 1.41 & 1.00 & 1.70 & 2 & & 106 & 27 & 1.44 & 0.80 & 1.60 & 3 \\
\hline & 1.09 & 26 & 1.42 & 1.00 & 1.71 & 2 & & 109 & 27 & 1.47 & 0.80 & 1.60 & 3 \\
\hline & 1.12 & 21 & 1.42 & 1.00 & 1.72 & 2 & & 112 & 27 & 1.50 & 0.80 & 1.60 & 3 \\
\hline & 1.15 & 20 & 1.43 & 1.00 & 1.73 & 2 & & 115 & 27 & 1.53 & 0.80 & 1.60 & 3 \\
\hline
\end{tabular}

costs. Only increases in $\mu_{1}$ produce more significant changes. An increase of $6 \%$ in $\mu_{1}$ results in a decrease in $h$ to 2 and increases in the values of $W^{0}$ and $C^{0}$, respectively, to 2.0 and 3.4.

With these observations, it is possible to notice that the optimum policy is reasonably robust in the range of variation of approximately $\pm 15 \%$ for the input parameters of the models. This evidence is important because the estimation of the parameters is not free of errors. 
The optimum solution presented above is not influenced by the values of $\phi_{0}=370$ and $\phi_{1}=5$, which are chosen by managers responsible for the production process because the non-restricted solution of the expression (10) [that is, $m^{0}=27 ; h^{0}=3 ; W^{0}=1.6, C^{0}=3.2, \$ 1.381$ ] presents values of $A R L_{0}=408.17$ and $A R L_{\varepsilon}=4.87$, which satisfy the two restrictions $\left(\phi_{0}=370\right.$ and $\phi_{1}=5$ ). However, the solution may be altered depending on the values chosen for $\phi_{0}$ and $\phi_{1}$. Table 2 shows the results for some values of $\phi_{0}$ and $\phi_{1}$. An increase in $\phi_{0}$ (keeping $\phi_{1}$ constant) tends to increase the optimum interval sampling $m$ while the values of $h, W$ and $C$ remain unaltered. An increase in $\phi_{1}$ does not alter significantly the results; however, when both $\phi_{0}$ and $\phi_{1}$ increase simultaneously, a considerable increase is observed in the sampling interval $m$. The value of $m$ changes from 27 to 193 . As expected, the cost increases as more demanding values of $\phi_{0}$ and $\phi_{1}$ are fixed.

Table 2 -Optimum parameters in the functions $\phi_{0}$ and $\phi_{1}$.

\begin{tabular}{|c|c|c|c|c|c|c|c|c|}
\hline$\phi_{0}$ & $\phi_{1}$ & $m$ & $h$ & $w$ & $c$ & cost & $A R L_{0}$ & $A R L_{\varepsilon}$ \\
\hline 370 & 5.00 & 27 & 3 & 0.8 & 1.6 & 1.381 & 408.173 & 4.872 \\
440 & 5.00 & 123 & 3 & 0.8 & 1.6 & 1.770 & 440.290 & 4.880 \\
370 & 4.85 & 28 & 2 & 1.0 & 1.7 & 1.382 & 392.751 & 4.877 \\
440 & 4.85 & 193 & 2 & 1.0 & 1.7 & 1.990 & 440.260 & 4.840 \\
\hline
\end{tabular}

The results presented in Table 1 are very useful, but they do not help us to indicate easily which parameters have a greater impact on the cost function. Identification of such parameters is very important because the parameter values are not free of errors, and those parameters that produce more impact on the cost need more (special) attention. For that, we use a full factorial experiment with the cost as the response and the following factors: $X_{1}$ - the cost to inspect $\left(c_{i}\right), X_{2}-$ the cost to send a non-conforming item $\left(c_{n}\right) ; X_{3}$ - the cost to scrap an inspected item $\left(c_{D}\right), X_{4}$ - the cost for adjustment $\left(c_{a}\right), X_{5}$ - the process mean when the process is out-of-control $\left(\mu_{1}\right)$ and $X_{6}$ - the probability for a shift in the process mean $(\pi)$. Three levels for each factor are chosen; the value described in the beginning of this section is set as a reference level, and two other values are fixed at $\pm 15 \%$ from the reference level. Such design results in 729 possible combinations, and a multiple regression model with dummy variables will be employed. For each factor, two dummy variables are defined, as follows:

$$
\left(X_{j 1}, X_{j 2}\right)=\left\{\begin{array}{l}
(0,1) \text { for } 1^{\text {st }} \text { level } \\
(1,0) \text { for } 2^{\text {nd }} \text { level } \\
(-1,-1) \text { for } 3^{\text {rd }} \text { level }
\end{array}\right.
$$

for $j=1, \ldots, 6$. The choice of which level will be the first, second or third level will not alter the results. Estimates of the coefficients $\beta_{0}, \beta_{j 1}, \beta_{j 2}, j=1, \ldots, 6$ of the regression model are obtained by the well-known least squares method. The range of the estimates of the coefficients (known as utilities) for each factor was the metric employed to evaluate the impact of each factor on the cost function. For more details, see Green \& Srinivasan (1990) and Malhotra (1999). For example, the estimate of the coefficient (utility) of the first level of 
the factor $j$ is $\hat{\beta}_{j 1}$, for the second level $\hat{\beta}_{j 2}$ and for the third level $\left(-\hat{\beta}_{j 1}-\hat{\beta}_{j 2}\right)$, with $\hat{\beta}_{j 1}$ and $\hat{\beta}_{j 2}$ obtained by the least squares method. The impact of factor $j$ is proportional to the range $\left[\max \left(\hat{\beta}_{j 1}, \hat{\beta}_{j 2},-\hat{\beta}_{j 1},-\hat{\beta}_{j 2}\right)\right]$ when compared with the range of the estimates of the coefficients from the other factors. Using this method, the impact of each factor is obtained and summarized in Table 3. Note that the factors $X_{4}$, the cost for adjustment $\left(c_{a}\right)$, and $X_{6}$, the probability for a shift in the process mean $(\pi)$, yield higher impacts, $31.3 \%$ and $35.1 \%$, respectively. Thus, these factors require careful evaluation and estimation. Some descriptive statistics may help to understand these results. In Figure 4, boxplots of each factor are drawn. Observe the upward tendency of the medians of the factors pointed out as more important while the medians of the other factors are kept constant when the levels alter.

Table 3 - The relative impact of the factors.

\begin{tabular}{|c|c|}
\hline Factor & Importance \\
\hline$c_{n}$ & $13.8 \%$ \\
$c_{D}$ & $2.6 \%$ \\
$\mu_{1}$ & $16.9 \%$ \\
$c_{a}$ & $31.3 \%$ \\
$c_{i}$ & $0.3 \%$ \\
$\pi$ & $35.1 \%$ \\
\hline
\end{tabular}
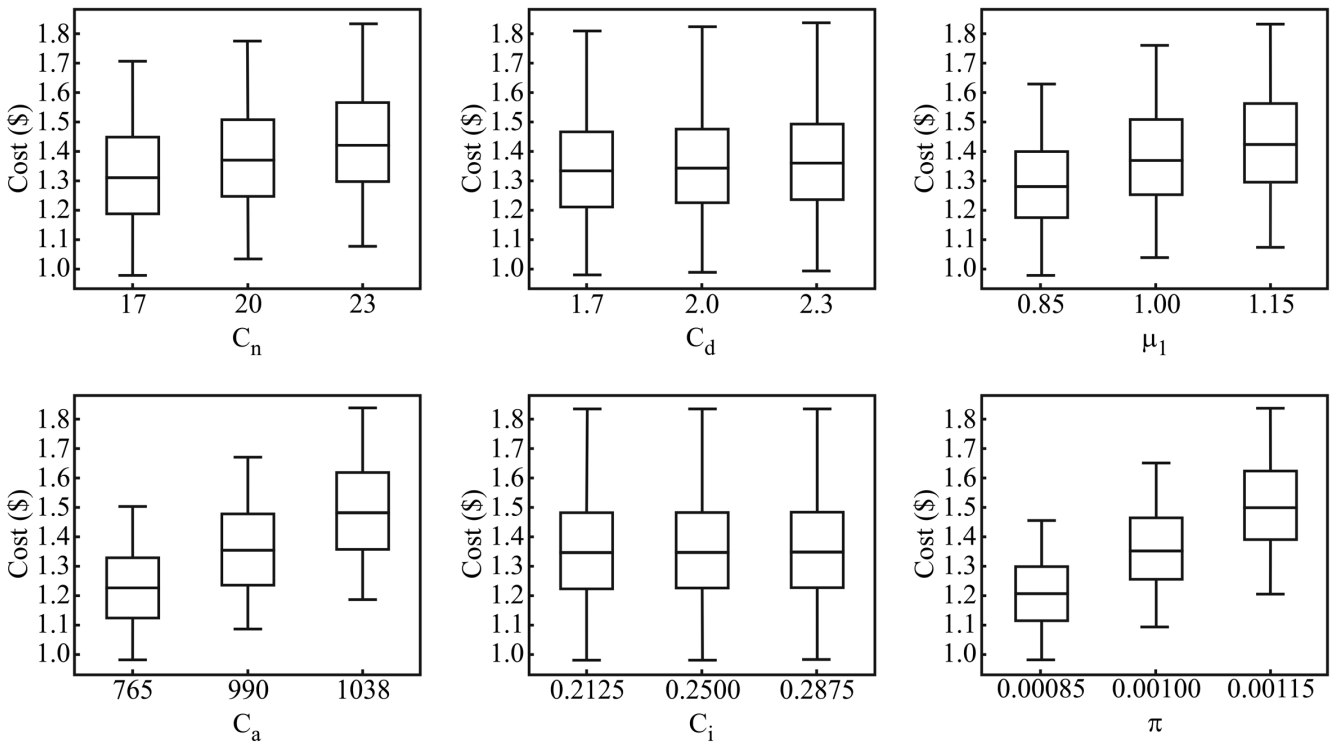

Figure 4 - BoxPlots of the factors $c_{I}, c_{n}, c_{S}, c_{a}, \mu_{1}$ and $\pi$. 


\section{CONCLUSIONS}

In earlier papers related to this subject, the optimum corrective policy designs for Taguchi's on-line quality monitoring procedure for variables are presented, and the sampling interval and the width of the control limits are fixed. In Ho, Medeiros \& Borges (2007), the authors determine the optimal sampling interval $m$ and the optimal control limit that minimizes the expected cost function.

In this paper, we extend the system of control by employing control zones. Three zones are stated: the green $\left(\mu_{0}-W \leq X_{i} \leq \mu_{0}+W\right)$, yellow $\left(\mu_{0}+W \leq X_{i}<\mu_{0}+C\right.$ or $\mu_{0}-C<$ $\left.X_{i} \leq \mu_{0}-W\right)$ and red $\left(X_{i}>\mu_{0}+C\right.$ or $\left.X_{i}<\mu_{0}-C\right)$. An observed value in the green zone results in the decision that the process is in-control and the production goes on; however, one decides in favor of an adjustment of the process if the observed value belongs to the red zone; moreover, a sequential verification takes place if an observation is in the yellow zone. If a sequence of $h$ values in the yellow zone is observed, then an adjustment is decided on.

The aim is to find the values of $m, h, W$ and $C$ that minimize the average cost. According to the results presented in Section 4, the inspection control and policy presented in this paper may yield results that are at least equal to or more economical than the results obtained by Ho, Medeiros \& Borges (2007).

Future research can be proposed. For example, the number of zones may increase. There is a tendency to have more automation in process control, so more complex alternatives may not produce excessive complexity for the user. The corrective adjustment based on $h$ sequential observations in the yellow zone $\left(\mu_{0}+W \leq X_{i}<\mu_{0}+C\right.$ or $\left.\mu_{0}-C<X_{i} \leq \mu_{0}-W\right)$, may be modified. For example Rule A: only $h$ sequential observations in the warning region above the centerline $\mu_{0}+W \leq X_{i}<\mu_{0}+C$; Rule B only $h$ sequential observations in the warning region below the centerline. Fixed $h$ and the warning limit $W$, Rule A (applied in this study) is more likely (higher probability) than Rule B when the process is out-of-control. Otherhand, Rule $\mathrm{B}$ is less unlikely (lower probability) than Rule A when the process is in-control. Preliminary simulation study conducted by the authors suggest that rules A and B present similar performance under an economical perspective once the optimum parameters $h, C$ and $W$ are different for each rule with similar average cost. However, a wider research may be realized to obtain better conclusions. Another possibility is to consider different sizes of sampling intervals. A longer sampling interval may be chosen for the next inspection if the current observation falls in the green zone or a shorter sampling interval may be chosen in the next inspection if the current point falls in the yellow zone. Other distributions for the process failure mechanism can be developed. A natural candidate is a discrete Weibull distribution [Nakagawa \& Osaki (1975)] because it can take on a variety of shapes and mimic the behavior of other distributions. Compared with a geometric distribution, the discrete Weibull distribution is more flexible in that it includes models with an increasing or decreasing hazard rate. 


\section{ACKNOWLEDGMENTS}

The authors would like to thank the suggestions from the two anonymous referees which have greatly contributed to the improvement of this paper. They would like to acknowledge CNPq and Fapesp for their partial financial support of this research.

\section{REFERENCES}

[1] Adams BM \& Woodall WH. 1989. An analysis of Taguchi's on-line process control procedure under a random-walk model. Technometrics, 31: 401-413.

[2] Borges W, Ho LL \& Turnes O. 2001. An analysis of Taguchi's on-line quality monitoring procedure for attributes with diagnosis errors. Applied Stochastic Models in Business and Industry, 17: $261-276$.

[3] Dasgupta T \& Mandal A. 2008. Estimation of process parameters to determine the optimum dagnosis interval for control of defective items. Technometrics, 50: 167-181.

[4] Ding J \& Gong L. 2008. The effect of testing equipment shift on optimal decisions in a repetitive testing process. European Journal of Operational Research, 186: 330-350.

[5] GReEn PE \& SRINIVASAN V. 1990. Conjoint analysis in marketing: new developments with implications for research and practice. Journal of Marketing, 54: 3-19.

[6] Ho LL, Medeiros PG \& Borges W. 2007. An alternative model for on-line quality monitoring for variables. International Journal of Production Economics, 107: 202-222.

[7] JIANG W \& TSUI KL. 2000. An economic model for integrated APC and SPC control charts. IIE Transactions, 32: 505-513.

[8] Malhotra NK. 1999. Marketing research: an applied orientation. Prentice-Hall. Inc.

[9] NANDi SN \& SReehari M. 1999. Some improvements in Taguchi's economic method allowing continued quality deterioration in production process. Communications in Statistics - Theory and Methods, 28(5): 1169-1181.

[10] Nakagawa T \& Osaki S. 1975. The Discrete Weibull Distribution. IEEE Transactions on Reliability, R-24 5: 300-301.

[11] Nayebrour MR \& Woodall WH. 1993. An analysis of Taguchi's on-line quality monitoring procedure for attributes. Technometrics, 35: 53-60.

[12] SRIVASTAVA MS \& WU Y. 1995. An improved version of Taguchi's on-line control procedure. Journal of Statistical Planning and Inference, 43: 133-145.

[13] SRIVAstaVa MS \& WU Y. 1991. A second order approximation on Taguchi's on-line procedures. Communications in Statistics - Theory and Methods, 20: 2149-2168.

[14] TAguchi G. 1985. Quality Engineering in Japan. Communications in Statistics - Theory and Methods, 14: 2785-2801.

[15] Taguchi G, Chowdhury S \& Wu Y. 2004. Taguchi's Quality Engineering - Handbook. John Wiley \& Sons, Inc. New Jersey.

[16] Taguchi G, Elsayed EA \& Hsiang T. 1989. Quality Engineering in Production Systems. McGraw-Hill, New York. 
[17] TRIndade A, Ho LL \& Quinino R. 2007. Monitoring process for attributes with quality deterioration and diagnosis errors. Applied Stochastic Models in Business and Industry, 23(4): 339-358.

[18] Western Electric Company. 1956. Statistical Quality Control Handbook, Western Eletric Company, Inc., Indianapolis, Indiana. 